Sainath Raman

Nigel Klein

Antonia Kwan

Mike Hubank

Shamima Rahman

Asrar Rashid

Mark J. Peters

\section{Oxidative phosphorylation gene expression falls at onset and throughout the development of meningococcal sepsis- induced multi-organ failure in children}

\section{Accepted: 9 April 2015}

Published online: 29 April 2015

(C) The Author(s) 2015. This article is published with open access at Springerlink.com

\section{Dear Editor,}

Sepsis-induced critical illness differs between adults and children. Children deteriorate more quickly and exhibit a 'cold shock' haemodynamic pattern more often. Organ failure scores characteristically peak earlier in septic children ( $<2$ ICU days) than adults (days 3-4). A high proportion of deaths in children occur very early [1]. Yet, ICU stays are shorter and survival better in children [2]. Might these differences in clinical phenotype-more rapid onset and recovery-be due to differences in the underlying mechanisms of multiorgan failure (MOF)?

Acute mitochondrial dysfunction may contribute to MOF. Reduced mitochondrial oxygen utilisation and gene expression has been observed in established sepsis in adults and children [3]. There is an association between recovery of mitochondrial function and survival but the contribution to the onset of organ failure is less clear. We investigated whether mitochondrial oxidative phosphorylation gene expression (Oxphos) alters early enough in the clinical course of sepsis to remain a candidate element of MOF pathophysiology. To do this, we selected a population with the most rapid onset of profound sepsis-MOF: previously healthy children with acute meningococcal septicaemia. We investigated the time-course of gene expression in peripheral blood with gene set enrichment analysis (GSEA), at 0,4 , $8,12,24$, and $48 \mathrm{~h}$ from time of admission to the emergency room.

Extracted RNA was hybridised in Affymetrix microarrays. Methodological details are published elsewhere [4]. The dataset is available to download from the European Bioinformatics Institute database (ArrayExpress id: E-MEXP-3850). Emergency room venesection was designated time 0 as a pragmatic

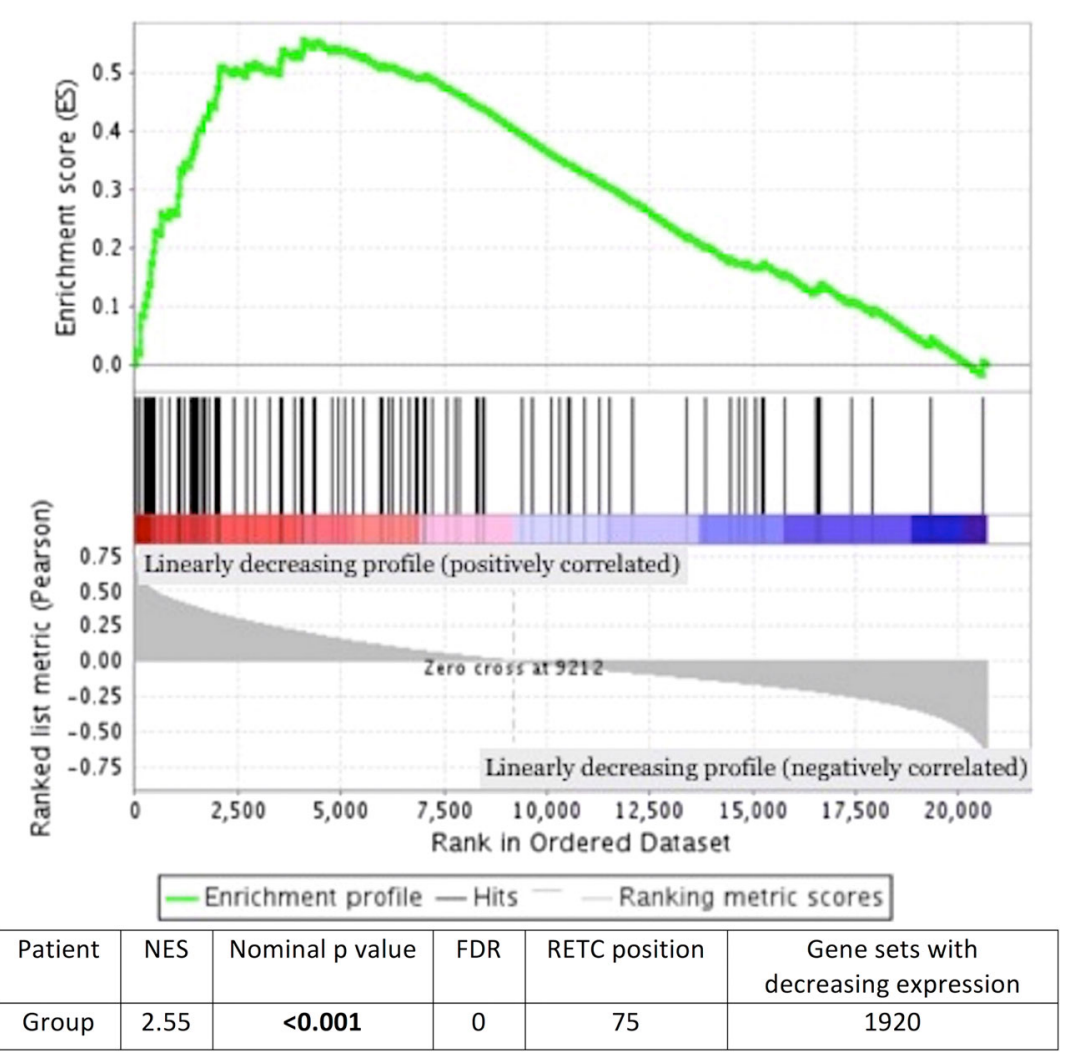

Fig. 1 Enrichment plot of the Respiratory Electron Transport Chain (RETC) gene set showing the normalised enrichment score $(N E S)$, the false discovery rate $(F D R)$ and nominal $p$ values for the gene set. Gene set enrichment analysis compares the variation in the gene expression in our overall dataset compared to a preselected gene set (RETC gene set) over the time-course. We have observed that RETC gene set expression is downregulated more than would be expected by background variation in expression. The top portion of the plot shows the running enrichment score $(E S)$ for the RETC gene set. The middle portion of the plot shows where the members of the gene set appear in the ranked list of genes. The lower portion of the plot shows the value of the ranking metric as you move down the list of ranked genes. The ranking metric measures a gene's correlation with a phenotype. For our continuous phenotype (time series), a positive value indicates correlation with the phenotype profile (decreasing profile) 
reference time point while acknowledging that children may be at different stages of illness at presentation.

The GSEA ranks changes in single gene expression. It notes the distribution of elements of a predefined gene set in this overall ranked list. We focused on the Reactome TCA cycle and respiratory electron transport chain (RETC) genes within the 3655 included gene sets. GSEA describes the probability of a non-random distribution of RTEC elements within the ranked list [5].

On the group level analysis, with a false discovery rate (FDR) at $<25 \%$ and ranked according to their normalised enrichment score (NES), 1039 out of 3655 gene sets showed a decreasing profile with time. The RETC set was ranked 75 th of the 1039 gene sets.

On the individual-level analysis and FDR $<25 \%$, all five patients had a highly ranked fall in RETC set expression. Patient 1 showed a low correlation of RETC gene expression to a decreasing profile. This might be relevant as, unfortunately, this child died. Figure 1 shows the NES, FDR and nominal $p$ values for the RETC set for the individual patients and the overall group.

Oxidative phosphorylation gene expression reduced early and continued to decrease for at least $48 \mathrm{~h}$ in septic critically ill children. These findings are consistent with mitochondrial dysfunction contributing to the development of organ failure in both adults and children despite the differences in sepsis phenotype in these groups.
Acknowledgment We thank the GSEA team (Broad institute, MIT) for their help with the analysis.

Conflicts of interest The authors do not have any competing interests.

Open Access This article is distributed under the terms of the Creative Commons Attribution-NonCommercial 4.0 International License (http://creativecommons.org/licenses/ by-nc/4.0/), which permits any noncommercial use, distribution, and reproduction in any medium, provided you give appropriate credit to the original author(s) and the source, provide a link to the Creative Commons license, and indicate if changes were made.

\section{References}

1. Cvetkovic M, Lutman D, Ramnarayan $P$ et al (2015) Timing of death in children referred for intensive care with severe sepsis: implications for interventional studies. Pediatr Crit Care Med. doi: 10.1097/PCC.0000000000000385

2. Schlapbach LJ, Straney L, Alexander J et al (2015) Mortality related to invasive infections, sepsis, and septic shock in critically ill children in Australia and New Zealand, 2002-13: a multicentre retrospective cohort study. Lancet Infect Dis 15:46-54. doi: 10.1016/S1473-3099(14)71003-5

3. Weiss SL, Cvijanovich NZ, Allen GL et al (2014) Differential expression of the nuclear-encoded mitochondrial transcriptome in pediatric septic shock. Crit Care 18:623. doi: 10.1186/PREACCEPT2292040841290621

4. Kwan A, Hubank M, Rashid A et al (2013) Transcriptional instability during evolving sepsis may limit biomarker based risk stratification. PLoS ONE 8:e60501

5. Subramanian A, Tamayo P, Mootha VK et al (2005) Gene set enrichment analysis: a knowledge-based approach for interpreting genome-wide expression profiles. Proc Natl Acad Sci USA 102:15545-15550

S. Raman $(\bowtie) \cdot$ M. J. Peters

Respiratory, Critical Care and Anaesthesia Unit, University College London-Institute of Child Health, 30 Guilford Street, London WC1N 1EH, UK

e-mail: sainath.raman@ucl.ac.uk

M. J. Peters

e-mail: mark.peters@ucl.ac.uk

\section{N. Klein}

Infectious Diseases and Immunology, University College London-Institute of Child Health, 30 Guilford Street, London WC1N 1EH, UK

e-mail:n.klein@ucl.ac.uk

A. Kwan

Department of Pediatrics, University of California, San Francisco, CA, USA

e-mail: Antonia.Kwan@ucsf.edu

M. Hubank

Genetics and Genomic Medicine,

University College London-Institute of Child Health, 30 Guilford Street, London WC1N 1EH, UK

e-mail:m.hubank@ucl.ac.uk

\section{S. Rahman}

Genetics and Genomics Programme, University College London-Institute of Child Health, 30 Guilford Street, London WC1N 1EH, UK

\section{A. Rashid}

EMBRACE, Sheffield Children's Hospital, Western bank, Sheffield S10 2TH, UK e-mail: asrar@mac.com 